\title{
EPR Spectroscopy of Different Sol Concentration Synthesized Nanocrystalline-ZnO Thin Films
}

\author{
Manju Arora, ${ }^{1}$ Rayees A. Zargar, ${ }^{2}$ and S. D. Khan $^{3}$ \\ ${ }^{1}$ CSIR-National Physical Laboratory, Dr. K. S. Krishnan Road, New Delhi 110012, India \\ ${ }^{2}$ Department of Physics, Jamia Millia Islamia, New Delhi 110025, India \\ ${ }^{3}$ Department of Electronics, Post Graduate College Bemina, Srinagar 190018, India \\ Correspondence should be addressed to Manju Arora; marora@nplindia.org
}

Received 30 June 2015; Revised 16 September 2015; Accepted 26 October 2015

Academic Editor: Kam-Sing Wong

Copyright (C) 2015 Manju Arora et al. This is an open access article distributed under the Creative Commons Attribution License, which permits unrestricted use, distribution, and reproduction in any medium, provided the original work is properly cited.

\begin{abstract}
Nanocrystalline zinc oxide ( $\mathrm{nc}-\mathrm{ZnO}$ ) thin films were grown on p-type silicon substrate through spin coating by sol-gel process using different sol concentrations (10 wt.\%, $15 \mathrm{wt} . \%$, and $25 \mathrm{wt}$.\%). These films were characterized by high resolution nondestructive Xray diffraction (XRD), scanning electron microscopy (SEM) with energy dispersive X-ray analysis (EDS) attachment, and electron paramagnetic resonance (EPR) techniques to understand variations in structural, morphological, and oxygen vacancy with respect to sol concentration. The film surface morphology changes from nanowall to nanorods on increasing sol concentration. EPR spectra revealed the systematic variation from ferromagnetic to paramagnetic nature in these nc-ZnO films. The broad EPR resonance signal arising from the strong dipolar-dipolar interactions among impurity defects present in nc-ZnO film deposited from $10 \mathrm{wt} . \%$ sol has been observed and a single strong narrow resonance signal pertaining to oxygen vacancies is obtained in $25 \mathrm{wt}$. $\%$ sol derived $\mathrm{nc}-\mathrm{ZnO}$ film. The concentrations of impurity defects and oxygen vacancies are evaluated from EPR spectra, necessary for efficient optoelectronic devices development.
\end{abstract}

\section{Introduction}

$\mathrm{ZnO}$ is one of the most extensively explored nanomaterials of band gap energy $\sim 3.37 \mathrm{eV}$ with large exciton binding energy of $60 \mathrm{meV}$. The wide band gap of $\mathrm{ZnO}$ thin films encouraged their usage in the field of photonics and chemical sensors [1-5]. The unique combination of $\mathrm{ZnO}$ piezoelectric and semiconducting properties makes it a promising material for many technological applications. Zinc oxide films derived MEMS can be used in pressure transducers, accelerometers, acoustooptic devices, and bulk and surface acoustic wave devices. Its doped analogues have been found to be an excellent material for energy storage and conversion photonic devices. The large specific surface area, nontoxicity, biocompatibility, chemical stability, electrochemical activity, and high electron density characteristics of nanocrystalline(nc-) $\mathrm{ZnO}$ with variety of morphological structures have drawn attention of the researchers for in-depth investigations.
The thin films of $\mathrm{ZnO}$ are deposited by physical and chemical routes to produce different types of one-dimensional (1D) nanostructures. Among them, $\mathrm{ZnO}$ nanowall, nanowire, and nanorod morphologies have been investigated with a view to using them for next generation optoelectronic, sensor, and memory device applications.

The chemical stability of nc- $\mathrm{ZnO}$ film has made it a suitable alternative candidate for conducting tin oxide and indium tin oxide films whose electrical and optical properties degrade in hydrogen plasma. Hence, the synthesis and characterization of $\mathrm{nc}-\mathrm{ZnO}$ thin films have been an active area of research and development. Nanostructured $\mathrm{ZnO}$ thin films can be grown by different methods such as pulsed laser deposition, CVD, MOCVD, sputtering, electrochemical deposition, sol-gel, and spray pyrolysis [6-15]. In these investigations, nc-ZnO films were deposited on pretreated p-type $\mathrm{Si}(100)$ wafer via spin coating technique by using $10 \mathrm{wt} . \%, 15 \mathrm{wt} . \%$, and $25 \mathrm{wt} . \%$ zinc acetate sol concentration. 
This is a very easy and cost-effective process for the uniform deposition of the film over large area of desired composition and morphology.

The aim of the present studies was to deposit nc- $\mathrm{ZnO}$ thin films from different sol concentration and characterize their structural, morphological, optical, and paramagnetic defects/impurities variations by XRD, SEM, and EPR techniques. The sensitive and sophisticated EPR spectroscopy technique has been used for ascertaining the creation of paramagnetic nature defects in $\mathrm{ZnO}$ films and their variation with sol concentration.

\section{Experimental Measurements}

nc- $\mathrm{ZnO}$ thin films were deposited on preoxidized single side polished (SSP) [100] p-type silicon (Si) substrate through spin coating technique by different concentrations. Before $\mathrm{ZnO}$ film deposition, Si substrate was oxidized by boiling the wafer in $70 \% \mathrm{HNO}_{3}$ to form thin layer of $\mathrm{SiOH}$ for better adhesion with deposited film to stop peeling and shedding off the deposited film. AR grade zinc acetate $\left[\mathrm{Zn}\left(\mathrm{CH}_{3} \mathrm{COO}\right)_{2} \cdot 2 \mathrm{H}_{2} \mathrm{O}\right]$ with $10 \mathrm{wt} . \%, 15 \mathrm{wt} . \%$, and $25 \mathrm{wt} . \%$ concentration was used for sol preparation and to clear the turbidity of sol solutions diethanolamine was added dropwise. Preoxidized Si wafer with drops of sol was spin rotated at the rate of 3200 rotations per minute (rpm) in the first two coatings and then increased by $1000 \mathrm{rpm}$ after every coating to a maximum of $3800 \mathrm{rpm}$ in spin coating machine, followed by drying the sol coated Si wafer at $80^{\circ} \mathrm{C}$ and then annealing at $450^{\circ} \mathrm{C}$ for one hour. Ten to fifteen layers were deposited on the same $\mathrm{Si}$ substrate to obtain the workable thickness of $\mathrm{ZnO}$ film as per the requirement of different characterization techniques. The ellipsometric data exhibits the variation in thickness of film from 2000 to $2500 \AA$ depending upon the number of coatings.

As synthesized nc-ZnO films structural details were analyzed by Bruker AXS D8 Advance X-ray diffractometer using $\mathrm{CuK} \alpha$ radiation with inbuilt Diffracplus software, LEO440 SEM with EDS attachment, Nano Scope II from Digital Instrument Inc. investigations for atomic arrangement of a surface and E-line Century Varian make E-112 X-band CW EPR spectrometer for paramagnetic defect analysis. The films were mounted transition metal ions free spectroscopic grade quartz rods and then placed at the center of $\mathrm{TE}_{102}$ cavity to avoid RF field saturation. $100 \mathrm{kHz}$ frequency was used for modulating magnetic field and $10 \mathrm{~mW}$ microwave power was applied to avoid resonance signal saturation effects. 1,1Diphenyl 2-picrylhydrazyl (DPPH) was used as a standard reference material for $g$-value and spin concentration estimations.

\section{Results and Discussion}

Zinc oxide formation by sol-gel process using zinc acetate dihydrate as precursor material consists of three steps, namely, hydrolysis, condensation, and polycondensation. In Step (1): hydrolysis reaction: zinc acetate dihydrate on dissolving in double distilled water forms partially hydrolyzed (basic zinc acetate) and mostly in ionic state $\left(\mathrm{Zn}^{2+}\right.$ and acetate ion). The hydrolysis process is sensitive to the atmospheric relative humidity at ambient temperature which facilitates the formation of basic zinc acetate. In Step (2): this mixture solution on heating forms two molecules of $\mathrm{Zn}(\mathrm{OH})_{2}$. In Step (3): condensation/polycondensation reaction form chain of $\mathrm{Zn}-\mathrm{O}$ with terminal $\mathrm{OH}$ groups. These three steps are represented by the following chemical equations:

(1) Hydrolysis reaction takes place on dissolution of zinc acetate in water:

$$
\begin{aligned}
& 2 \mathrm{Zn}\left(\mathrm{CH}_{3} \mathrm{COO}\right)_{2}+\mathrm{H}_{2} \mathrm{O} \\
& \Longleftrightarrow \mathrm{Zn}(\mathrm{OH})\left(\mathrm{CH}_{3} \mathrm{COO}\right)+\mathrm{CH}_{3} \mathrm{COOH} \\
& \text { (basic zinc acetate) } \\
& +\mathrm{Zn}^{2+}+2 \mathrm{CH}_{3} \mathrm{COO}^{-} \\
& \text {(zinc ions and acetate ions) }
\end{aligned}
$$

(2) Ionized zinc acetate upon heating evaporates off zinc hydroxide and acetic acid while basic zinc acetate hydrolyzes to zinc hydroxide and acetate ions. These chemical reactions are given as

$$
\begin{gathered}
\mathrm{Zn}\left(\mathrm{CH}_{3} \mathrm{COO}\right)_{2}+2 \mathrm{H}_{2} \mathrm{O} \\
\longrightarrow \mathrm{Zn}(\mathrm{OH})_{2}+2 \mathrm{CH}_{3} \mathrm{COOH} \uparrow \\
\mathrm{Zn}(\mathrm{OH})\left(\mathrm{CH}_{3} \mathrm{COO}\right)+\mathrm{OH}^{-} \\
\longrightarrow \mathrm{Zn}(\mathrm{OH})_{2}+2 \mathrm{CH}_{3} \mathrm{COO}^{-}
\end{gathered}
$$

(3) Condensation/polycondensation reaction are given as

$$
\begin{aligned}
& \mathrm{HO}-\mathrm{Zn}-\mathrm{OH}+\mathrm{HO}-\mathrm{Zn}-\mathrm{OH} \\
& \longrightarrow \mathrm{HO}-\mathrm{Zn}-\mathrm{O}-\mathrm{Zn}-\mathrm{OH}+\mathrm{H}_{2} \mathrm{O}
\end{aligned}
$$

The polycondensation reaction is like a chain propagation reaction for $n$ number of molecules product; it can be expressed as

$$
\begin{gathered}
\mathrm{HO}-(\mathrm{Zn}-\mathrm{O}-\mathrm{Zn})_{n-1}-\mathrm{OH}+\mathrm{HO}-\mathrm{Zn}-\mathrm{OH} \\
\longrightarrow \underset{\text { (polycondensate with terminal hydroxyls })}{\mathrm{HO}-(\mathrm{Zn}-\mathrm{O}-\mathrm{Zn})_{n}-\mathrm{OH}+\mathrm{H}_{2} \mathrm{O}}
\end{gathered}
$$

The process continues till maximum chained stable polycondensate is sustained or the precursor exhausted in solution. After the evaporation of the water molecules, the final product can be written as $\mathrm{HO}-(\mathrm{Zn}-\mathrm{O}-\mathrm{Zn})_{n}-\mathrm{OH}$, where $n$ is the number of molecules taking part in the polycondensation process. This reaction spreads steadily and uniformly in all directions through crystallization process on the surface of substrate.

3.1. X-Ray Analysis. Zinc oxide crystallizes in hexagonal wurtzite structure with crystal lattice having alternate planes composed of tetrahedrally coordinated $\mathrm{O}^{2-}$ and $\mathrm{Zn}^{2+}$ ions, bonded along the $c$-axis $[16,17]$. 
TABLE 1: Structural parameters of nc-ZnO thin films derived from $10 \mathrm{wt} . \%, 15 \mathrm{wt} . \%$, and $25 \mathrm{wt} . \%$ sol concentration.

\begin{tabular}{lccccccc}
\hline nc-ZnO sol conc. & Plane & $d(\AA)$ & FWHM $(\beta)$ & $2 \theta$ & $D(\mathrm{~nm})$ & $\begin{array}{c}\delta \times 10^{-3} \\
(\mathrm{~nm})^{-2}\end{array}$ \\
\hline 10 wt.\% & 002 & 2.59402 & 0.06221 & 34.340 & 26.997 & 1.372 & $\begin{array}{c}\varepsilon \times 10^{-2} \\
15 \text { wt.\% }\end{array}$ \\
25 wt.\% & 002 & 2.59582 & 0.04695 & 34.340 & 35.766 & 0.7817 \\
\hline
\end{tabular}

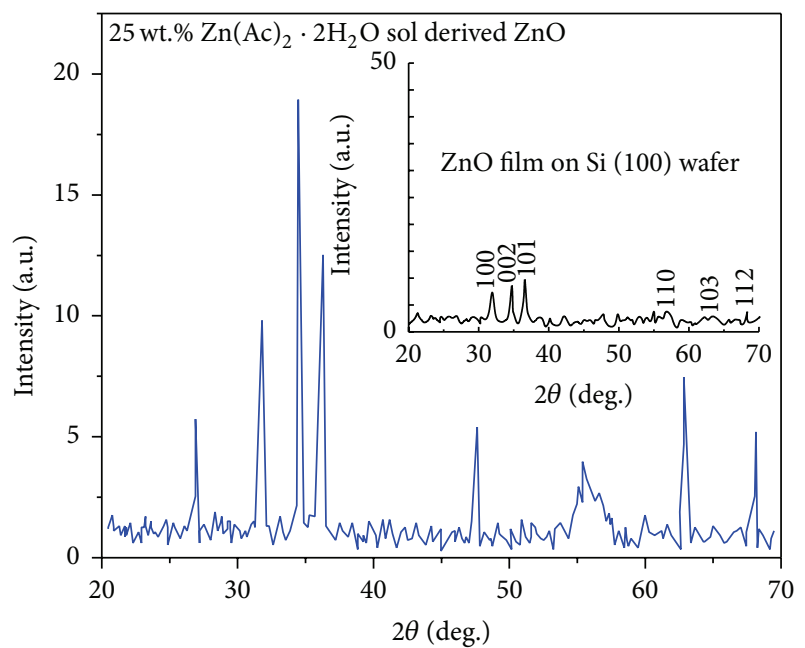

FIGURE 1: XRD patterns of $10 \%$ (inset) and $25 \%$ sol derived nc-ZnO thin film.

XRD pattern of $10 \mathrm{wt}$.\% sol concentration derived nc$\mathrm{ZnO}$ thin film is presented in Figure 1 (inset) which shows polycrystalline nature without any preferentially oriented plane present in the film. The diffraction peaks at $2 \theta$ (in degrees) are observed at 31.680, 34.340, 36.180, 56.540, 62.780, and 68.260 of [100], [002], [101], [110], and [112] planes, respectively, as marked in Figure 1. The unit cell dimensions " $a$ " and " $c$ " of the polycrystalline- $\mathrm{ZnO}$ films with (002) orientation are calculated using the following equations [18]:

$$
\begin{aligned}
& a=\frac{\sqrt{1 / 3} \lambda}{\sin \theta}, \\
& c=\frac{\lambda}{\sin \theta} .
\end{aligned}
$$

The calculated lattice unit cell dimensions for $a$ and $c$ are $0.3254( \pm 0.0004) \mathrm{nm}$ and $0.5212( \pm 0.0008) \mathrm{nm}$, respectively. These values are near to the reported values for $a$ and $c$, $0.3250 \mathrm{~nm}$ and $0.5207 \mathrm{~nm}$, respectively, listed in PDF \#36-1451 data card. On increasing sol concentration, it was found that the preferred orientation of [002] plane, that is, along the $c$ axis of film, improves. The crystallite size of $\mathrm{ZnO}$ thin film was measured by using Debye-Scherrer's formula:

$$
D=\frac{k \lambda}{\beta \cos \theta},
$$

where $D$ is crystallite size, $k$ is proportionality constant $(=0.9), \lambda$ is wavelength $1.5406 \AA$ for $\mathrm{CuK} \alpha$ X-ray radiation used, $\beta$ is the full width at half maximum (FWHM) of diffraction peak in radians, and $\theta$ is the Bragg angle. In these $10 \mathrm{wt} . \%, 15 \mathrm{wt} . \%$, and $25 \mathrm{wt} . \%$ sol concentrations grown nc$\mathrm{ZnO}$ thin films have crystallite size values calculated from [002] peak in the range of 25 to $45 \mathrm{~nm}$.

The dislocation density $(\delta)$ gives information about the amount of defects present in the crystal and is calculated from the following equation:

$$
\delta=\frac{1}{D^{2}}
$$

Strain $(\varepsilon)$ present in the thin film is determined from the following formula:

$$
\varepsilon=\frac{\beta \cos \theta}{4}
$$

The evaluated structural parameters derived from the XRD patterns are presented in Table 1.

From Table 1, it has been found that the crystallite size increases with the increase of precursor sol concentration. At lower precursor concentration, that is, $10 \mathrm{wt} . \%$, the nucleation rate is relatively slow, which may end up in small crystallite size [19]. But in higher sol concentrations, the crystallite improves due to increased growth in lateral features size with the reduction in dislocation density and strain. This indicates improvement in crystallinity of films and tendency towards high degree of agglomeration among smaller crystallites to form bigger agglomerated crystallites as evidenced from SEM images. Hence, the increase in crystallite size with sol concentration can also be associated with the shape and size of nanostructures present in $\mathrm{ZnO}$ films.

3.2. SEM and EDS Analysis. SEM micrographs of $10 \mathrm{wt} . \%$ and 25 wt.\% sol derived $\mathrm{ZnO}$ thin film are presented in Figures 2 (a) and 2(b), respectively, recorded at $\times 20000$ magnification of nanowall structure and at $\times 40000$ magnification. SEM micrograph of $10 \%$ sol derived nc- $\mathrm{ZnO}$ thin film exhibits the nanowalls growth and randomly patterned interwoven nanorods uniformly covering the surface of film as shown in Figures 2(a) and 2(b), respectively. These nanowalls are considered as a suitable candidate for field emission applications. SEM micrograph revealed the smoothness of the deposited film.

The initial random orientation of the nanograins in the nanowall morphology has been attributed to the poor crystallization in low $10 \mathrm{wt}$ \% sol concentration nc- $\mathrm{ZnO}$ film. When the sol concentration is increased to $25 \mathrm{wt} . \%$, the dipole-dipole interaction between polar grains encourages the formation of nanorod/spindle shape morphology and 


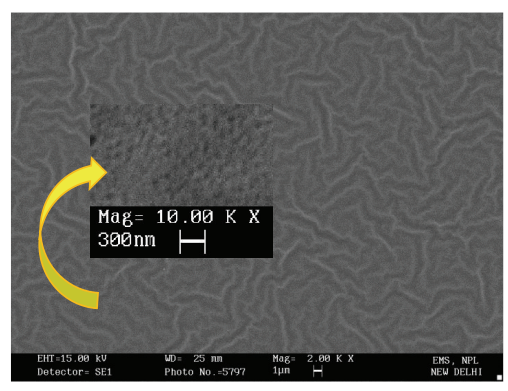

(a)

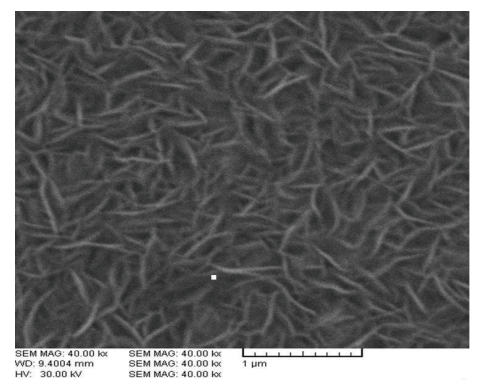

(b)

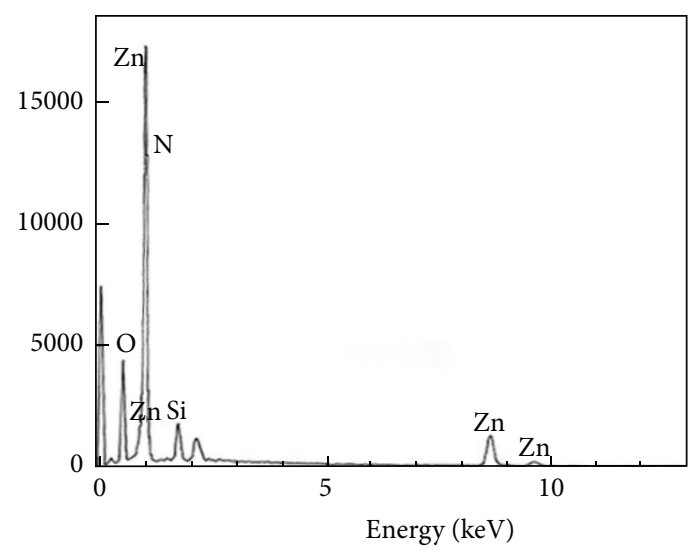

FIGURE 2: SEM micrograph: (a) $10 \mathrm{wt} . \%$ and (b) $25 \mathrm{wt} . \%$ sol derived nc-ZnO thin film and EDS of $25 \%$ sol derived nc-ZnO thin film.

orientation of nanograins along the $c$-axis, which improves the overall crystallinity of $\mathrm{ZnO}$ film. The similar results are also evidenced in XRD patterns of these films as shown in Figure 1. The EDS spectrum of 25 wt.\% sol concentration nc$\mathrm{ZnO}$ thin film exhibits strong intensity $\mathrm{Zn}$ and $\mathrm{O}$ peaks with weak intensity Si substrate peaks by $15 \mathrm{keV}$ incident electron for confirming the formation of $\mathrm{ZnO}$ stoichiometry. $\mathrm{ZnK} \alpha$ and $\mathrm{ZnK} \beta$ excitation peaks are observed at $8.64 \mathrm{keV}$ and $9.57 \mathrm{keV}$, respectively, and oxygen peak is also observed in EDS spectrum.

The atomic arrangement of the atoms present at surface of a thin film is studied by Scanning Tunneling Microscopy (STM) which detects the corrugations in the electron density on the surface of film. These corrugations are evolved from the positions of atoms on the surface. The three-dimensional profiles of different locations of the surface were generated to observe surface roughness, surface defects, and conformation of molecules and aggregates on the surface. As we know, that $\mathrm{ZnO}$ synthesis chemical reaction involves hydrolysis, condensation, and polycondensation of zinc hydroxide. During spin coating, the slip or dislocation in atomic arrangement of $\mathrm{ZnO}$ takes place more at the peripheries then in the center. The atomic arrangement in $100 \mu \mathrm{m}$ thick $25 \mathrm{wt} . \%$ sol concentration of $\mathrm{nc}-\mathrm{ZnO}$ thin film has been used for recording STM micrograph as shown in Figure 3 which exhibits arrays of alternate dark and bright areas of collection of $\mathrm{ZnO}$ atoms of oriented polar nanograins [20].

3.3. EPR Analysis. Electron paramagnetic resonance (EPR) spectroscopy is a very powerful sensitive technique used for the characterization of nature of magnetic impurities/defects and spin dynamics in materials that have paramagnetic defects [21-36] in nanocrystalline zinc oxide. EPR spectra are scanned by varying the magnetic field at constant microwave frequency (i.e., $9.36 \mathrm{GHz}$ in X-band EPR spectrometer). The magnetic field position of EPR resonance line depends upon the nature and environment around paramagnetic center free electron which affects the effective gyrometric factor ( $g$-value) and sensitivity for measuring spin concentrations
Scan distance $(80.01 \mu \mathrm{m})$

$Z$ distance $(2500.13 \mathrm{~nm})$

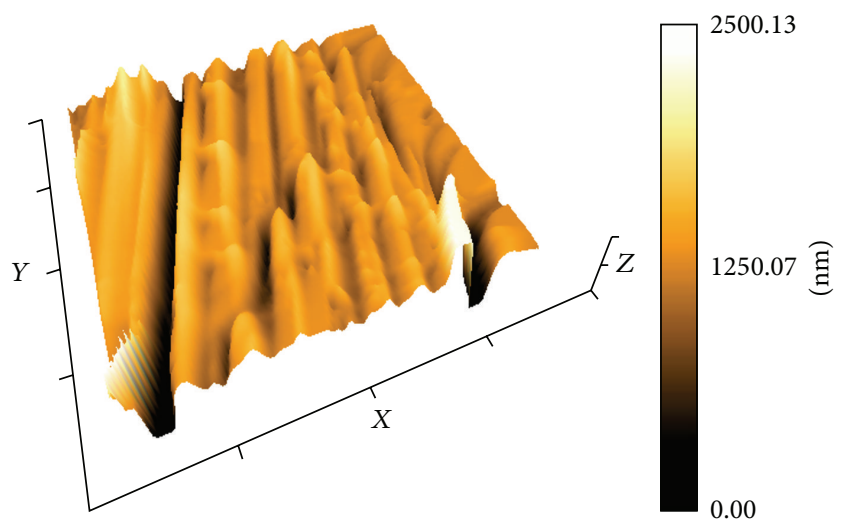

Figure 3: STM image of $25 \mathrm{wt} . \%$ sol derived nc-ZnO thin film.

from ppm to ppb level. EPR spectra as shown in Figure 4 of these nc- $\mathrm{ZnO}$ thin films vary from broad resonance signal to intermediate narrow signal superimposed on broad resonance signal to narrow single asymmetric resonance signal by varying sol concentration between low $10 \mathrm{wt} . \%$, intermediate 15 wt. $\%$, and 25 wt.\%, respectively. Earlier both experimental and theoretical studies reported the presence of neutral single charge and double charge zinc vacancies, neutral zinc interstitials, singly charged zinc interstitial, neutral oxygen vacancies, singly charged oxygen vacancy, oxygen interstitial, and complex of an oxygen vacancy and zinc interstitial type defects in $\mathrm{ZnO}$ [24-28, 34-36]. In bulk $\mathrm{ZnO}$, the electrons are delocalized at the defect centers and it is very difficult to saturate their transitions at room temperature and their EPR spectra are generally observed at low temperatures [23]. While in nc- $\mathrm{ZnO}$, electrons confine at defect sites and restrict their mobility. EPR signal is observed at ambient temperature resulting from low electric surface conductivity of isolated defects with relatively easy saturation effects and long spinspin relaxation time. 
TABLE 2: EPR parameters evaluated from $10 \mathrm{wt} . \%, 15 \mathrm{wt} . \%$, and $25 \mathrm{wt} . \%$ sol concentration of nc-ZnO thin films spectra.

\begin{tabular}{lcccc}
\hline Sample & $\begin{array}{c}\text { Peak-to-peak } \\
\text { linewidth } \Delta H_{\mathrm{PP}} \\
\text { (Gauss) }\end{array}$ & $g$-value & $\begin{array}{c}\text { Spin concentration } \\
N_{S}(\text { spin/g) }\end{array}$ & $\begin{array}{c}\text { Spin-spin relaxation } \\
\text { time constant } \\
T_{2}(\mathrm{~s})\end{array}$ \\
\hline $10 \mathrm{wt}$ \% sol & 1265.52 & 2.0034 & $2.276 \times 10^{20}$ & $4.2138 \times 10^{-15}$ \\
$15 \mathrm{wt}$ \% sol & 65.27 & 1.9673 & $1.573 \times 10^{18}$ & $1.5137 \times 10^{-13}$ \\
$25 \mathrm{wt}$ \% sol & 159.42 & 1.9682 & $3.728 \times 10^{18}$ & $3.2642 \times 10^{-13}$ \\
\hline
\end{tabular}

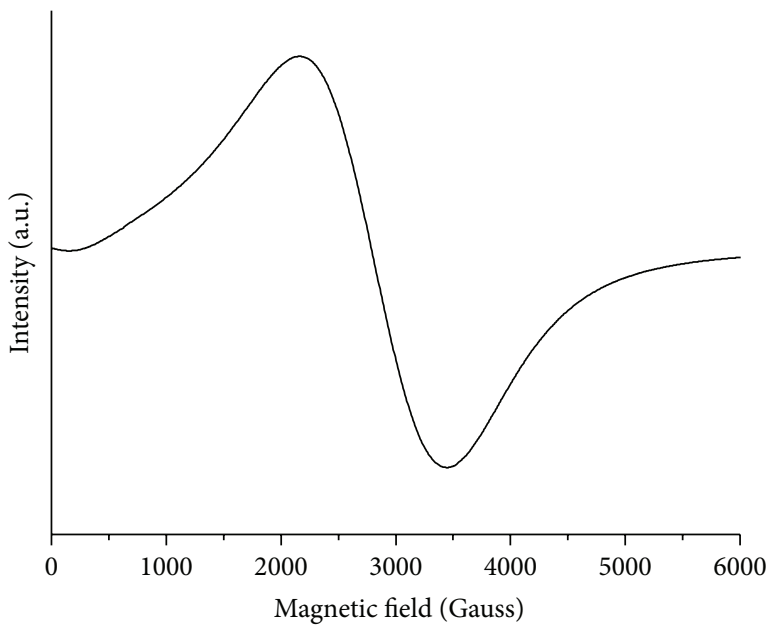

(a)

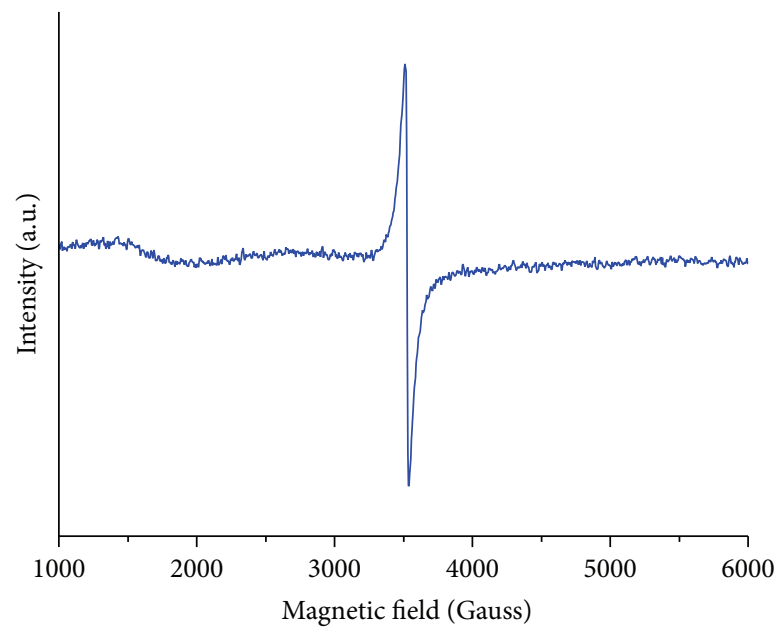

(c)

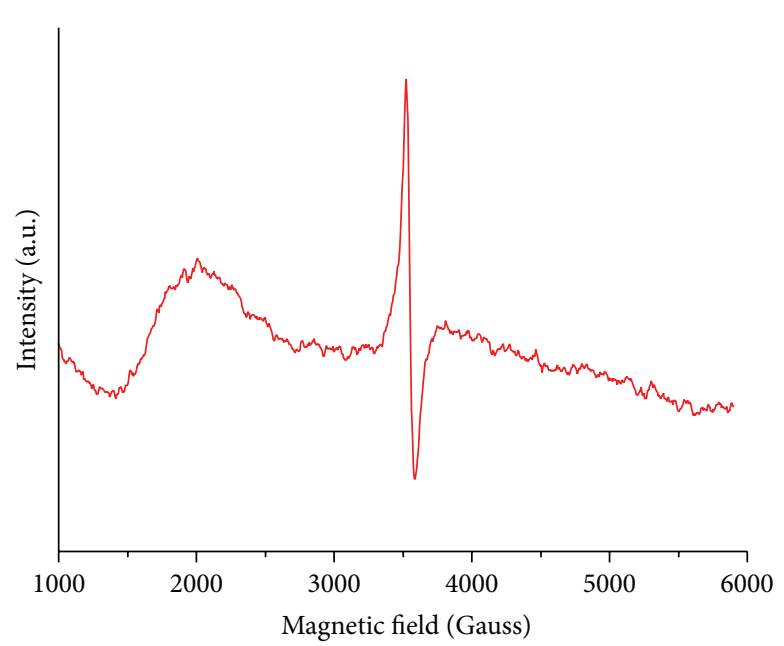

(b)

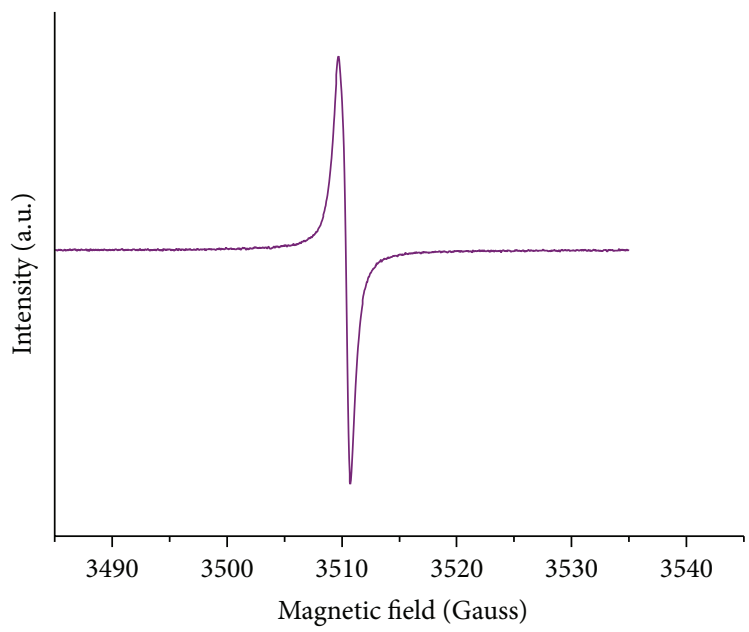

(d)

FIGURE 4: EPR spectra of nc-ZnO thin films derived from (a) 10 wt.\%, (b) 15 wt.\%, and (c) 25 wt. \% sol and (d) DPPH standard.

All the spectra obtained were analyzed using Lorentzian distribution function to obtain the values of various EPR parameters such as $\Delta H_{\mathrm{PP}}, g$-value, $N_{x}$, and $T_{2}$ and the values are listed in Table 2. The linewidth and shape of resonant signal give information about the magnetic and electronic state. The broad resonance signal exhibits the ferromagnetic nature of defects which are produced in lattice by excessive $\mathrm{Zn}$ vacancies related shallow donors [24, 26, 27, 29, 30, 36]. The broadness of the EPR resonance signal is due to strong dipolar-dipolar interactions among randomly oriented shallow defects in low sol concentration film, which scatter in the directions of anisotropic field of the film. In high sol content sample, the decrease in linewidth is attributed to the isotropic alignment of magnetic moments with increase in oxygen vacancy concentration $[21-23,25,28,33-36]$. The $g$-value is basically a proportionality constant between frequency and magnetic field and its value is sensitive to molecular motion, paramagnetic properties, and the symmetry of ions [31]. $g$-value is calculated by the relation $E=h v=g \beta H$, $g=h v / \beta H$, where $h$ is Planck constant, $v$ is the microwave frequency, $\beta$ is Bohr magneton, and $H$ is the magnetic field at resonance. In these nc- $\mathrm{ZnO}$ thin films, $g$-value varies with 
increase in zinc acetate sol concentration, which exhibits the strengthening of magnetic moment with increase in oxygen vacancy concentration.

The spin concentration is calculated by the comparison method. In this method, the spins concentration in unknown sample is measured with respect to standard DPPH sample of known spin concentration which nullifies instrumental and environmental errors by recording EPR spectra of sample and standard reference sample under the same conditions. The spin concentration of paramagnetic centers present in unknown sample is calculated from the expression given as

$$
N_{x}=\frac{A_{x}\left(\operatorname{Scan}_{x}\right)^{2} G_{s} M_{s}\left(g_{s}\right)^{2}\left[S(S+1)_{s}\right] N_{s}}{A_{s}\left(\operatorname{Scan}_{s}\right)^{2} G_{x} M_{x}\left(g_{x}\right)^{2}\left[S(S+1)_{x}\right]},
$$

where subscripts $s$ and $x$ represent the standard and unknown sample, respectively, $A$ is area measured under absorption curve, $M$ is modulation amplitude, $G$ is the relative gain of signal amplifier, Scan is horizontal scale in Gauss per unit length, $S$ is the spin number, and $g$ is the $g$-factor of EPR signal. In this expression area under the curve and mass of sample are key parameters in spin concentration calculations. It follows the similar trend as observed in peak-to-peak linewidth of these films.

The spin relaxation process deals with release of excited spin excessive energy to the surrounding spins and within lattice. EPR spectrum can be used to find the value of spinspin relaxation time constant as represented the following equation:

$$
\begin{aligned}
\frac{1}{T_{2}} & =\left(g \beta \Delta H_{1 / 2}\right), \\
\Delta H_{1 / 2} & =\sqrt{3} \Delta H_{\mathrm{PP}},
\end{aligned}
$$

where $g$ is $g$-value, $\beta$ is Bohr magneton, and $\Delta H_{1 / 2}$ is constant, that is, linewidth at half of the absorption peak. In these films, the relaxation time decreases with increase in sol wt.\%.

As the sol concentration increases to $15 \mathrm{wt} . \%$, the appearance of narrow signal superimposed on broad resonance signal confirms the coexistence of zinc vacancies related defects and oxygen vacancies present in film lattice. While in $25 \mathrm{wt}$ \% sol derived nc-ZnO thin film, a narrow asymmetrical resonance signal of $g$-value 1.9682 arising from oxygen vacancies is observed. Oxygen vacancies were formed during film heating process and sophisticated nondestructive EPR spectroscopy technique has been successfully used for the characterization and concentration evaluation of such defects. This $g$-value of bulk $\mathrm{ZnO}$ is 1.9456 and the increase in $g$-value of these films is explained in terms of the quantum size effect in nanocrystalline materials due to confined electron movement which can easily saturate [36].

\section{Conclusions}

In these investigations, nanocrystalline zinc oxide thin films were grown on silicon substrate by sol-gel method with different sol concentrations. XRD, SEM, EDS, STM, and
EPR studies revealed the uniform, polycrystalline, hexagonal structure preferentially orientated along the $c$-axis. In 10 wt.\% sol concentration $\mathrm{ZnO}$ film, nanowalls morphology is observed due to nucleation of nanoparticles in wall type pattern. The broad signal due to excessive $\mathrm{Zn}$ vacancies in $10 \mathrm{wt} . \%$ low sol concentration nc- $\mathrm{ZnO}$ film exhibits the ferromagnetic behavior and narrow signal superimposed on broad resonance signal reflects the intermediate state of the $15 \%$ sol derived thin film in which zinc and oxygen vacancies coexist in the film lattice. While in $25 \%$ sol derived nc- $\mathrm{ZnO}$ thin film, a narrow line resonance signal of $g$-value 1.9682 is attributed to the presence of paramagnetic oxygen vacancies only in higher sol concentration samples. The increase in $g$-value as compared to bulk $\mathrm{ZnO}$ is attributed to the quantum confinement effect of nanorods. The paramagnetic oxygen vacancies are formed in these films during growth process and present successful use of nondestructive EPR spectroscopy technique for such type of studies.

\section{Conflict of Interests}

The authors declare that there is no conflict of interests regarding the publication of this paper.

\section{References}

[1] L. A. Patil, A. R. Bari, M. D. Shinde, and V. Deo, "Ultrasonically prepared nanocrystalline $\mathrm{ZnO}$ thin films for highly sensitive LPG sensing," Sensors and Actuators, B: Chemical, vol. 149, no. 1, pp. 79-86, 2010.

[2] P. Bhattacharyya, P. K. Basu, B. Mondal, and H. Saha, "A low power MEMS gas sensor based on nanocrystalline $\mathrm{ZnO}$ thin films for sensing methane," Microelectronics Reliability, vol. 48, no. 11-12, pp. 1772-1779, 2008.

[3] Z. L. Wang, "Zinc oxide nanostructures: growth, properties and applications," Journal of Physics Condensed Matter, vol. 16, no. 25, pp. R829-R858, 2004.

[4] M. Giannouli and F. Spiliopoulou, "Effects of the morphology of nanostructured $\mathrm{ZnO}$ films on the efficiency of dye-sensitized solar cells," Renewable Energy, vol. 41, pp. 115-122, 2012.

[5] B. Bayraktaroglu, K. Leedy, and R. Neidhard, "Nanocrystalline $\mathrm{ZnO}$ microwave thin film transistors," in Micro- and Nanotechnology Sensors, Systems, and Applications II, vol. 7679 of Proceedings of SPIE, pp. 1024-1026, Orlando, Fla, USA, May 2010.

[6] J. R. Casanova, E. A. Heredia, C. D. Bojorge, H. R. Cánepa, G. Kellermann, and A. F. Craievich, "Structural characterization of supported nanocrystalline $\mathrm{ZnO}$ thin films prepared by dipcoating," Applied Surface Science, vol. 257, no. 23, pp. 1004510051, 2011.

[7] C. Shaoqiang, Z. Jian, F. Xiao et al., "Nanocrystalline ZnO thin films on porous silicon/silicon substrates obtained by sol-gel technique," Applied Surface Science, vol. 241, no. 3-4, pp. 384391, 2005.

[8] S. Venkatachalam, Y. Kanno, and S. Velumani, "Characterization on pulsed laser deposited nanocrystalline $\mathrm{ZnO}$ thin films," Vacuum, vol. 84, no. 10, pp. 1199-1203, 2010.

[9] S. T. Tan, B. J. Chen, X. W. Sun et al., "Blueshift of optical band gap in $\mathrm{ZnO}$ thin films grown by metal-organic chemical-vapor 
deposition," Journal of Applied Physics, vol. 98, no. 1, Article ID 013505, 5 pages, 2005.

[10] Y. L. Liu, Y. C. Liu, Y. X. Liu et al., "Structural and optical properties of nanocrystalline $\mathrm{ZnO}$ films grown by cathodic electrodeposition on Si substrates," Physica B: Condensed Matter, vol. 322, no. 1-2, pp. 31-36, 2002.

[11] T. Sahoo, M. Kim, M.-H. Lee et al., "Nanocrystalline $\mathrm{ZnO}$ thin films by spin coating-pyrolysis method," Journal of Alloys and Compounds, vol. 491, no. 1-2, pp. 308-313, 2010.

[12] N. L. Tarwal, V. V. Shinde, A. S. Kamble et al., "Photoluminescence and photoelectrochemical properties of nanocrystalline $\mathrm{ZnO}$ thin films synthesized by spray pyrolysis technique," Applied Surface Science, vol. 257, no. 24, pp. 10789-10794, 2011.

[13] V. Srikant and D. R. Clarke, "Anomalous behavior of the optical band gap of nanocrystalline zinc oxide thin films," Journal of Materials Research, vol. 12, no. 6, pp. 1425-1428, 1997.

[14] M. Dutta, S. Mridha, and D. Basak, "Effect of sol concentration on the properties of $\mathrm{ZnO}$ thin films prepared by sol-gel technique," Applied Surface Science, vol. 254, no. 9, pp. 27432747, 2008.

[15] L. Znaidi, G. J. A. A. Soler Illia, S. Benyahia, C. Sanchez, and A. V. Kanaev, "Oriented $\mathrm{ZnO}$ thin films synthesis by sol-gel process for laser application," Thin Solid Films, vol. 428, no. 1-2, pp. 257262, 2003.

[16] M. Ristić, S. Musić, M. Ivanda, and S. Popović, "Sol-gel synthesis and characterization of nanocrystalline $\mathrm{ZnO}$ powders," Journal of Alloys and Compounds, vol. 397, no. 1-2, pp. L1-L4, 2005.

[17] S. J. Pearton, D. P. Norton, K. Ip, Y. W. Heo, and T. Steiner, "Recent progress in processing and properties of $\mathrm{ZnO}$," Progress in Materials Science, vol. 50, no. 3, pp. 293-340, 2005.

[18] C. Suryanarayana, C. Norton, and M. Grant, X-Ray Diffraction: A Practical Approach, Plenum Press, New York, NY, USA, 1998.

[19] X. Zhao, J. Y. Lee, C.-R. Kim et al., "Dependence of the properties of hydrothermally grown $\mathrm{ZnO}$ on precursor concentration," Physica E, vol. 41, no. 8, pp. 1423-1426, 2009.

[20] J. Wang, Y. Qi, Z. Zhi, J. Guo, M. Li, and Y. Zhang, "A selfassembly mechanism for sol-gel derived $\mathrm{ZnO}$ thin films," Smart Materials and Structures, vol. 16, no. 6, pp. 2673-2679, 2007.

[21] M. Arora, S. K. Gupta, H. Bahadur, and S. Chandra, "EPR spectroscopic study of nanocrystalline $\mathrm{ZnO}$ thin films," in Proceedings of the 52nd DAE Solid State Physics Symposium, pp. 261-262, Mysore, India, December 2007.

[22] A. K. Srivastava, Praveen, M. Arora et al., "Nanostructural features and optical performance of RF magnetron sputtered $\mathrm{ZnO}$ thin films," Journal of Materials Science and Technology, vol. 26, no. 11, pp. 986-990, 2010.

[23] H. Zhou, A. Hofstaetter, D. M. Hofmann, and B. K. Meyer, "Magnetic resonance studies on $\mathrm{ZnO}$ nanocrystals," Microelectronic Engineering, vol. 66, no. 1-4, pp. 59-64, 2003.

[24] P. H. Kasai, "Electron spin resonance studies of donors and acceptors in ZnO," Physical Review, vol. 130, no. 3, pp. 989-995, 1963.

[25] A. Hausmann and B. Schallenberger, "Interstitial oxygen in zinc oxide single crystals," Zeitschrift für Physik B Condensed Matter, vol. 31, no. 3, pp. 269-273, 1978.

[26] S. Greulich-Weber, S. J. Azam, C. Brodehl, and T. Baumgarten, "Surface-related defects of $\mathrm{ZnO}$ micro and nano crystals prepared by sol-gel technique," Physica Status Solidi, vol. 10, no. 2, pp. 221-226, 2013.

[27] S. G. Weber, S. A. Casas, and N. Weber, "Low temperature nanocrystalline zinc oxide for photovoltaic applications," IOP
Conference Series: Materials Science and Engineering, vol. 80, Article ID 012009, 6 pages, 2015.

[28] V. A. Nikitenko, K. E. Tarkpea, I. V. Pykanov, and S. G. Stoyukhin, "EPR and thermo-luminescence in $\mathrm{ZnO}$ single crystals with ionic vacancies," Journal of Applied Spectroscopy, vol. 68, no. 3, pp. 502-507, 2001.

[29] D. Gao, Z. Zhang, J. Fu, Y. Xu, J. Qi, and D. Xue, "Room temperature ferromagnetism of pure $\mathrm{ZnO}$ nanoparticles," Journal of Applied Physics, vol. 105, no. 11, Article ID 113928, 5 pages, 2009.

[30] X. Zuo, S.-D. Yoon, A. Yang, W.-H. Duan, C. Vittoria, and V. G. Harris, "Ferromagnetism in pure wurtzite zinc oxide," Journal of Applied Physics, vol. 105, no. 7, Article ID 07C508, 2009.

[31] J. A. Weil, J. R. Bolton, and J. E. Wertz, Electron Paramagnetic Resonance-Elementary Theory and Practical Applications, Wiley, New York, NY, USA, 1994.

[32] R. A. Zargar, M. Arora, and A. K. Hafiz, "Investigation of physical properties of screen printed nanosized $\mathrm{ZnO}$ films for optoelectronic applications," The European Physical Journal Applied Physics, vol. 70, no. 1, Article ID 10403, 6 pages, 2015.

[33] W. E. Carlos, E. R. Glaser, and D. C. Look, "Magnetic resonance studies of ZnO," Physica B, vol. 308-310, pp. 976-979, 2001.

[34] L. S. Vlasenko, "Magnetic resonance studies of intrinsic Defects in ZnO: oxygen vacancy," Applied Magnetic Resonance, vol. 39, no. 1, pp. 103-111, 2010.

[35] V. Ischenko, S. Polarz, D. Grote, V. Stavarache, K. Fink, and M. Driess, "Zinc oxide nanoparticles with defects," Advanced Functional Materials, vol. 15, no. 12, pp. 1945-1954, 2005.

[36] P. Jakes and E. Erdem, "Finite size effects in $\mathrm{ZnO}$ nanoparticles: An electron paramagnetic resonance (EPR) analysis," Physica Status Solidi-Rapid Research Letters, vol. 5, no. 2, pp. 56-58, 2011. 

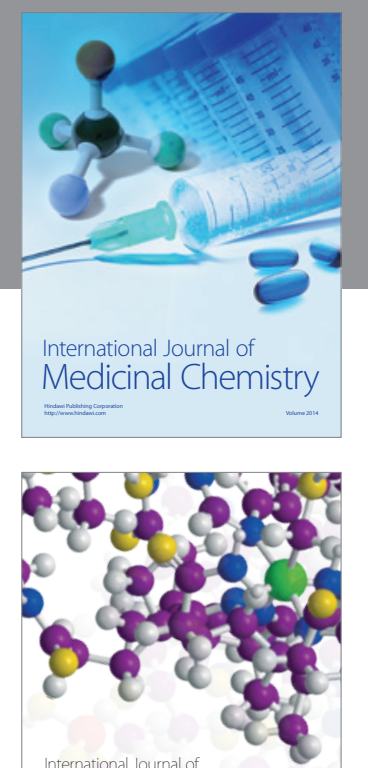

\section{Carbohydrate} Chemistry

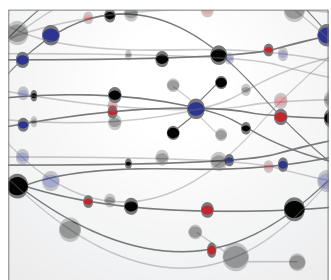

The Scientific World Journal
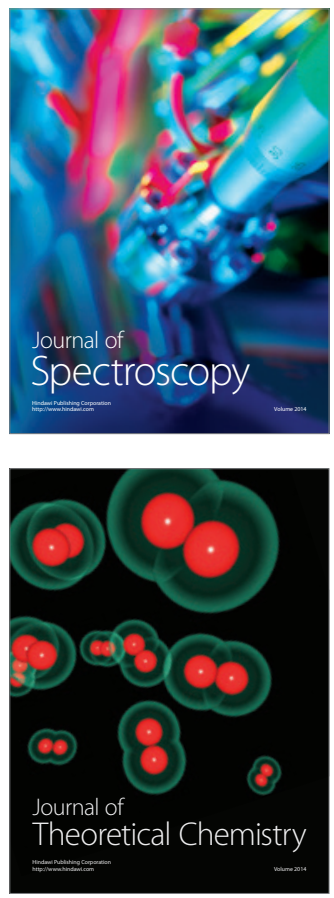
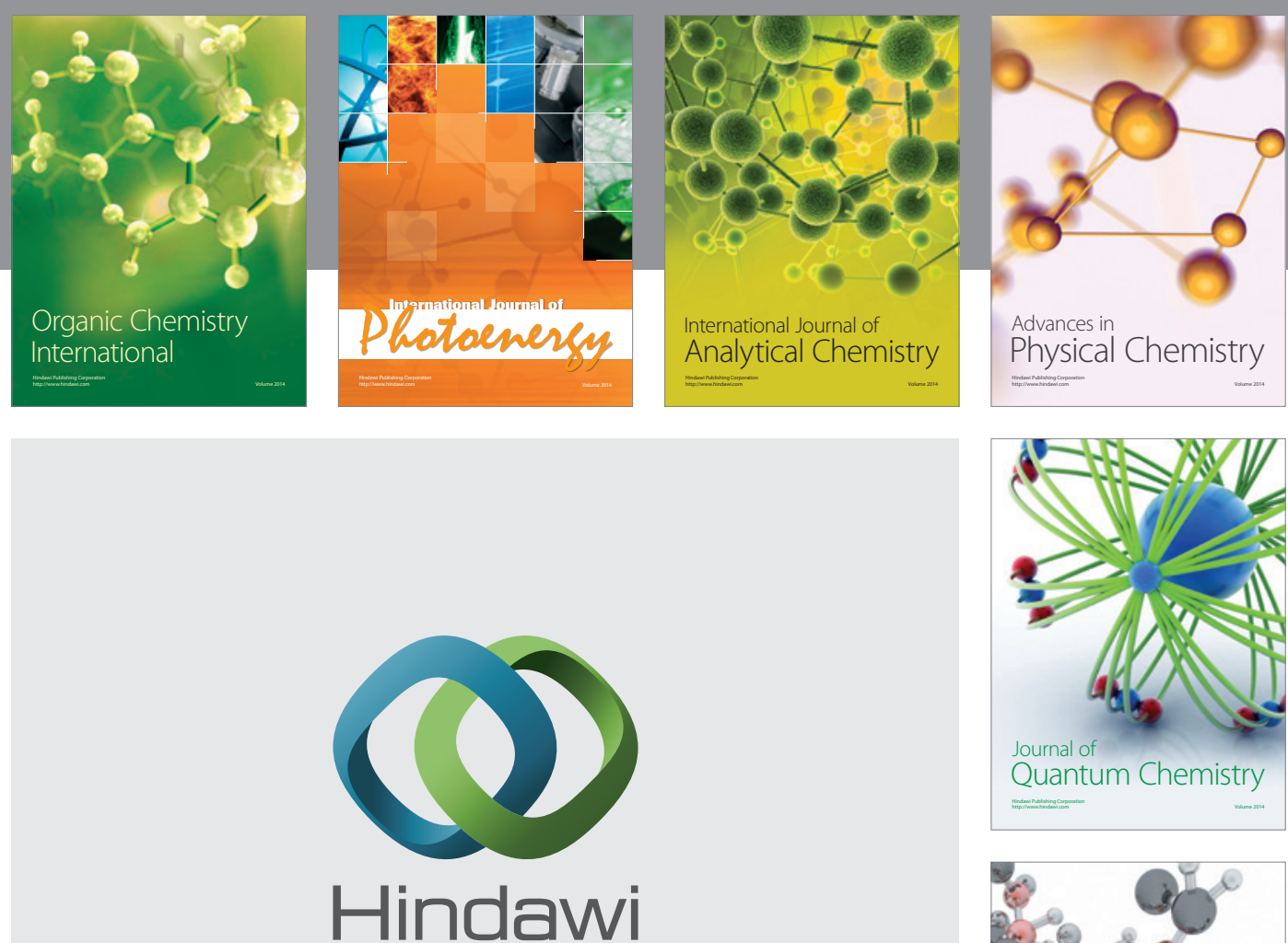

Submit your manuscripts at

http://www.hindawi.com

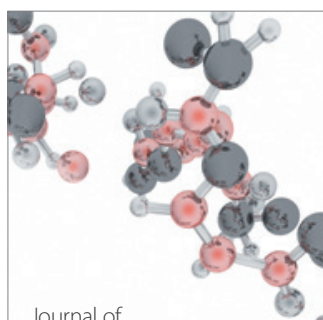

Analytical Methods

in Chemistry

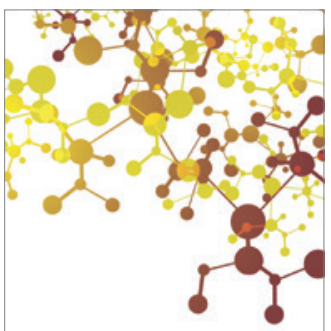

Journal of

Applied Chemistry

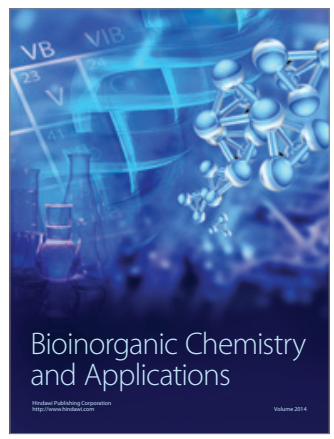

Inorganic Chemistry
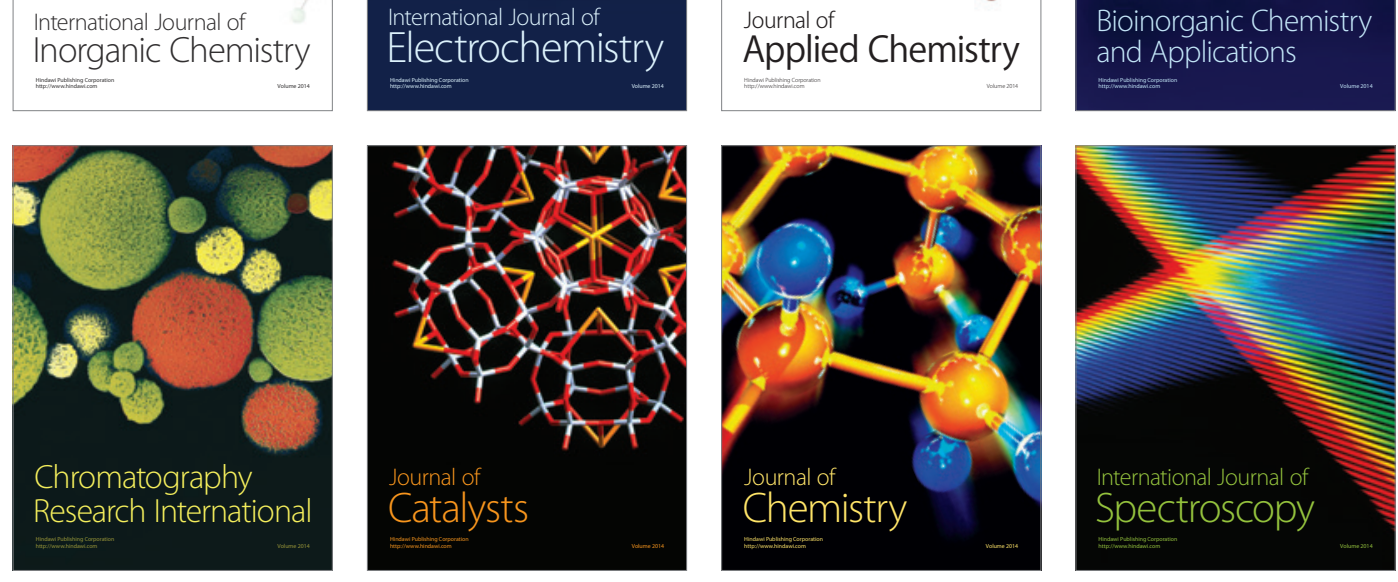